\title{
Caracterización de materiales de maracuyá (Passiflora spp) en Veracruz, México
}

Material characterization of passion fruit (Passiflora spp) in Veracruz, México

Becerra Leor Enrique N. ${ }^{\boxplus}$, Xochítl Rosas-González ${ }^{1}$, Isaac Meneses-Márquez ${ }^{1}$ y Andrés VásquezHernández ${ }^{1}$

${ }^{1}$ INIFAP-Campo Experimental Cotaxtla Km 34.5 Carretera federal Veracruz-Córdoba. Municipio de Medellín de Bravo, Ver.

${ }^{\bowtie}$ Autor para correspondencia: becerra.noe@inifap.gob.mx

Recibido: 09/01/2014
Aceptado: 19/07/2014

\section{RESUMEN}

Se llevó a cabo la caracterización de diferentes materiales de maracuyá incluyendo tres especies cultivadas ( $P$. edulis Deg, $P$. ligularis Juss y $P$. quadrangularis L.) y una silvestre ( $P$. foetida L. Gaertn). El análisis de varianza mostro diferencias altamente significativas entre materiales y hubo correlación entre el contenido jugo y la cantidad de semillas por fruto, y además entre jugo y peso del fruto. $P$. quadrangularis presentó los frutos más grandes y $P$. foetida los de menor tamaño.

Palabras claves: Evaluación, passifloras, especies

\begin{abstract}
Characterization of different materials of passion fruit including three cultivated species ( $P$. edulis Sims fv flavicarpa Deg., and P. ligularis Juss. P. quadrangularis L.) and a wild (P. foetida L. Gaertn.) was carried out. Analysis of variance showed significant differences among materials broadly and there were correlation between juice content and number of seeds per fruit, and juice content with fruit weight. $P$. quadrangularis had the largest fruit than others and P. foetida the smaller one.
\end{abstract}

Keywords: Evaluation, passifloras, species.

\section{INTRODUCCION}

El maracuyá es uno de los nuevos cultivos que se ha introducido para su siembra en las áreas tropicales y subtropicales, en un intento por diversificar la fruticultura. Este fruto es originario de la región del Amazonas en Brasil, que es el país con mayor producción en el mundo. Otros países productores importantes son: Colombia, Ecuador, Perú, Australia, Sudáfrica, y Estados Unidos (Hawaii y Florida); también se cultiva en Venezuela, República Dominicana, El Salvador, Costa Rica, Puerto Rico, Cuba, Chile y México,

Revista Científica Biológico Agropecuaria Tuxpan 2 (1) 
aunque en este último país se produce en baja escala (Schwentesius y Gómez, 1997; Knight y Sauls, 1994).

Los grandes países productores de maracuyá como Brasil, Venezuela y Ecuador, no tienen identificadas variedades, únicamente lo conocen como maracuyá amarillo. En El Salvador es un cultivo relativamente nuevo, originado de semilla introducida de Guatemala y posiblemente de Honduras; algunos productores tienen identificado su material, con base en una selección propia, pero posiblemente no saben su verdadero nombre (García, 2002).

En los Estados Unidos (California, Hawaii y Massachussets) se han desarrollado variedades de maracuyá morado como Black Knight, Edgehill, Frederick, Kahuna, Paul Ecke y Purple Giant (Morton, 1987).

De maracuyá amarillo se mencionan a Brazilian Golden y Golden Giant, de Brasil y de Australia, respectivamente. Existen algunas selecciones locales como Waimanalo Selection, Yee Selection y Noel's Special de Hawai, además de Mirim o Hawaiiana de América del Sur. En Australia se siembran híbridos de las dos formas como E 23, Purple Gold, Lacey y Black Beauty. En Brasil se han obtenido recientemente tres híbridos denominados BRS Gigante Amarelo, BRS Ouro Vermelho y BRS Sol do Cerrado (EMBRAPA, 2008).

En México este cultivo se produce en baja escala, la superficie es pequeña, los lotes de producción son reducidos en la mayoría de los casos y las prácticas agronómicas y uso de materiales mejorados son empíricas. Veracruz cuenta con las condiciones agro-ecológicas que satisfacen los requerimientos del cultivo. El objetivo de este trabajo fue caracterizar y seleccionar germoplasma para contribuir a la diversificación de genotipos de este cultivo.

\section{MATERIALES Y METODOS}

El experimento se realizó en lotes del Campo Experimental Cotaxtla, del INIFAP, ubicado en el kilómetro 34.5 de la carretera Veracruz-Córdoba, en el municipio de Medellín de Bravo. Se evaluaron 16 materiales de maracuyá durante el periodo de febrero a mayo del 2008 y 2009. Se consideraron materiales de $P$. edulis, $P$. ligularis, $P$. foetida y $P$. quadrangularis, de los cuales nueve son maracuyá amarillo, cuatro morados, dos rosadas y una anaranjada (Cuadro 2). Se midieron los siguientes datos en diez frutos de cada material: Peso en gramos (g), diámetro a lo largo y ancho fruto, largo y ancho de la cavidad en centímetros $(\mathrm{cm})$, número de semillas, relación largo y ancho del fruto, grosor de cáscara en milímetros $(\mathrm{mm}), \mathrm{pH}$ y color de la pulpa, sólidos solubles, contenido de jugo (ml) y en algunos casos acidez titulable. Se hicieron análisis de varianza y correlaciones con el paquete estadístico SAS.

\section{RESULTADOS Y DISCUSION}

En el Cuadro 1 se presentan los resultados del análisis de varianza de los materiales en donde se obtuvieron diferencias altamente significativas para los parámetros de jugo expresado en mililitros, peso del fruto expresado en gramos, número de semillas, cantidad de sólidos solubles, relación largo por ancho del fruto y $\mathrm{pH}$ del jugo.

El material AmarilloB sobresalió en los siguientes parámetros peso de fruto, largo y ancho de cavidad de fruto, numero de semillas y jugo (238 gr; $8.68 \mathrm{~cm} ; 8.11 \mathrm{~cm} ; 328.7$ y $93.9 \mathrm{ml}$, respectivamente). También sobresalieron Cotaxtla en peso promedio (128 gr); y largo y ancho de la cavidad el material de La Esperanza $(15.76 \mathrm{~cm}$ y $30.35 \mathrm{~cm} ; 7.55 \mathrm{~cm}$ y $5.82 \mathrm{~cm}$

Revista Científica Biológico Agropecuaria Tuxpan 2 (1)

ISSN: 2007-6940 
respectivamente), en grosor de cáscara casi acidez titulable fue de 3.55 a 4.98 .

todos los materiales contaron con $0.34 \mathrm{~mm}$; la

Cuadro 1. Cuadrados medios de variables con diferencias altamente significativas de maracuyá.

\begin{tabular}{|c|c|c|c|c|c|c|c|}
\hline F.V. & G.L. & Jugo $(\mathrm{ml})$ & $\begin{array}{c}\text { Peso fruto } \\
(\mathrm{g})\end{array}$ & No. Semillas & $\begin{array}{c}\text { Solidos } \\
\text { Solubles }\end{array}$ & $\begin{array}{c}\text { Relaciòn } \\
\text { Largo/Ancho }\end{array}$ & Ph \\
\hline Rep & 4 & 204.3 & 755.1 & 4525 & 4.6 & 0.003 & 0.03 \\
\hline Variedad & 15 & $1165.4 * *$ & $8323.7 * *$ & $14,774.4 * *$ & $5.9 * *$ & $0.48 * *$ & $0.95 * *$ \\
\hline Error & 60 & 16.4 & 64.4 & 579.5 & 0.3 & 0.0003 & 0.001 \\
\hline & & & & & & & \\
\hline C.V. & & 9.4 & 6 & 9.8 & 3.6 & 2.2 & 1 \\
\hline
\end{tabular}

Los materiales morados sobresalieron en los sólidos solubles con promedios de 15.4 a 18.4 (Cuadro 2). La acidez titulable vario de
3.61 a 4.43. El color de la pulpa varió desde amarillo hasta anaranjado.

Cuadro 2. Comparaciones de medias de variables evaluadas en materiales de maracuyá.

\begin{tabular}{|c|c|c|c|c|c|c|c|c|c|c|}
\hline Variedad & \multicolumn{2}{|c|}{ peso/fruto(g) } & \multicolumn{2}{|c|}{ Num. de semilla } & \multicolumn{2}{|c|}{ Brix } & \multicolumn{2}{|c|}{ Indice (largo/an } & \multicolumn{2}{|l|}{$\mathrm{pH}$} \\
\hline Amarilla J1 & 156.1 & $\mathrm{~b}$ & 253.8 & efg & 15.0 & efgh & 0.95 & $\mathrm{f}$ & 3.0 & $\mathrm{~d}$ \\
\hline Morada J & 49.0 & 1 & 124.0 & o & 14.9 & efghi & 0.88 & $\mathrm{~g}$ & 3.2 & $\mathrm{c}$ \\
\hline Rosada J1 & 150.8 & $\mathrm{~b}$ & 292.1 & $\mathrm{~cd}$ & 16.1 & bc & 0.52 & $\mathrm{i}$ & 3.4 & $\mathrm{~b}$ \\
\hline Morada CECOT & 128.0 & cde & 246.8 & efgh & 14.6 & fghijk & 0.52 & $\mathrm{i}$ & 2.8 & fg \\
\hline Morada redonda Rancho El Para & 97.6 & hi & 201.0 & ijklmn & 15.4 & cde & 1.06 & $\mathrm{e}$ & 2.8 & $\mathrm{~h}$ \\
\hline Rosada Rancho El Paraiso & 100.4 & hi & 234.8 & fghi & 13.9 & $\mathrm{k}$ & 1.14 & $\mathrm{c}$ & 2.7 & $\mathrm{i}$ \\
\hline Amarilla Jalisco & 105.6 & $\mathrm{~h}$ & 219.8 & $\begin{array}{c}\text { ghijkl } \\
\text { m }\end{array}$ & 16.0 & bcd & 0.52 & $\mathrm{i}$ & 3.0 & d \\
\hline Amarilla CECOT & 128.0 & cdef & 327.4 & $a b$ & 16.3 & $\mathrm{~b}$ & 0.53 & $\mathrm{i}$ & 2.7 & $\mathrm{i}$ \\
\hline Amarilla B & 238.3 & a & 328.7 & a & 14.8 & efghij & 0.86 & $\mathrm{~g}$ & 3.0 & $\mathrm{e}$ \\
\hline Amarilla La Esperanza & 123.9 & defg & 223.5 & fghijkl & 15.3 & def & 0.64 & $\mathrm{i}$ & 3.0 & d \\
\hline Amarilla Comapa & 129.6 & cde & 258.1 & ef & 15.2 & defg & 0.65 & $\mathrm{~h}$ & 2.9 & $\mathrm{f}$ \\
\hline Amarilla San Martinito & 122.4 & defg & 226.0 & fghijk & 14.2 & ijk & 0.52 & $\mathrm{i}$ & 2.8 & fg \\
\hline Amarilla Rancho Paraiso & 137.4 & $\mathrm{c}$ & 302.8 & $\mathrm{bc}$ & 16.1 & $\mathrm{bc}$ & 1.09 & de & 2.8 & $\mathrm{~h}$ \\
\hline Amarilla Paso La Mipla & 129.8 & $\mathrm{~cd}$ & 279.4 & cde & 14.6 & fghijk & 1.38 & a & 3.1 & $\mathrm{c}$ \\
\hline Granadilla Chiapas & 77.6 & $\mathrm{j}$ & 231.8 & fghij & 14.4 & hijk & 1.34 & $\mathrm{~b}$ & 4.5 & a \\
\hline Morada T & 63.6 & $\mathrm{k}$ & 172.8 & $\mathrm{n}$ & 18.4 & $\mathrm{a}$ & 1.15 & $\mathrm{c}$ & 2.9 & e \\
\hline Significancia & $* *$ & & $*$ & $*$ & & $* *$ & $* *$ & & $* *$ & \\
\hline
\end{tabular}

La correlación entre el contenido de jugo $(\mathrm{ml})$ y el peso del fruto entre variedades resultó altamente significativo $\left(\mathrm{R}^{2}=0.7\right)($ Fig.
1). Lo mismo sucedió cuando se correlacionó entre contenido de jugo $(\mathrm{ml})$ y número de semillas $\left(\mathrm{R}^{2}=0.5\right)($ Figura 2$)$. Lo anterior es 
importante porque se buscaria producir frutos con mayor cantidad de semilla, lo que permitiria obtener una buena cantidad de jugo

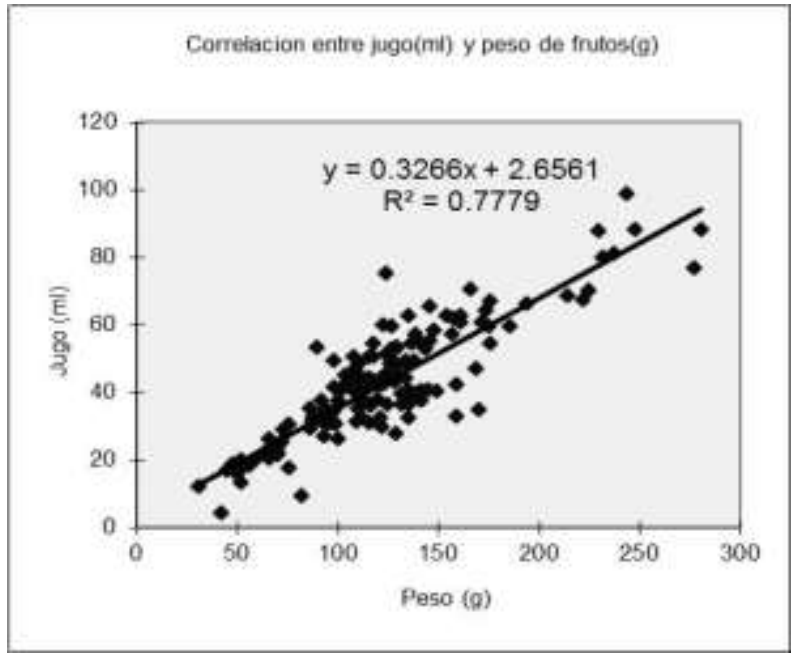

Figura 1. Correlación significativa entre jugo y peso del fruto. por fruto. Sobretodo que la produccion de este cultivo se enfoca a la industria de los jugos.

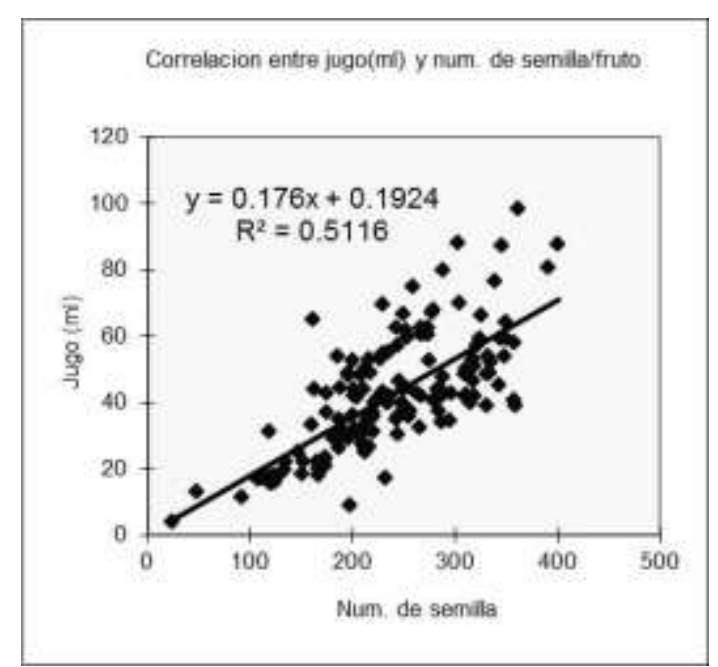

Figura 2. Correlación entre jugo y número de semillas.

Cuadro 3. Características morfológicas de especies de Passiflora

\begin{tabular}{|c|c|c|c|c|c|c|c|c|c|c|c|c|}
\hline \multirow{2}{*}{$\begin{array}{l}\text { Especies de } \\
\text { Passifloras }\end{array}$} & \multirow{2}{*}{$\begin{array}{l}\text { Peso de } \\
\text { fruto }(\mathrm{g})\end{array}$} & \multirow{2}{*}{$\begin{array}{l}\text { Largo } \\
\text { fruto } \\
(\mathrm{cm}) \\
\end{array}$} & \multirow{2}{*}{$\begin{array}{l}\text { Ancho } \\
\text { fruto } \\
(\mathrm{cm}) \\
\end{array}$} & \multicolumn{2}{|c|}{ Cavidad (cm) } & \multirow{2}{*}{$\begin{array}{c}\text { No. } \\
\text { semillas }\end{array}$} & \multirow{2}{*}{$\begin{array}{c}\text { Grosor } \\
\text { cascara } \\
(\mathrm{mm})\end{array}$} & \multirow{2}{*}{$\begin{array}{l}\text { pH de } \\
\text { pulpa }\end{array}$} & \multirow{2}{*}{$\begin{array}{c}\text { Color de } \\
\text { pulpa }\end{array}$} & \multirow{2}{*}{$\begin{array}{l}\text { Sólidos } \\
\text { solubles }\end{array}$} & \multirow{2}{*}{$\begin{array}{l}\text { Jugo } \\
(\mathrm{ml})\end{array}$} & \multirow{2}{*}{$\begin{array}{l}\text { Acidez } \\
\text { titulable }\end{array}$} \\
\hline & & & & Largo & Ancho & & & & & & & \\
\hline P. foetida & 3.2 & 2.5 & 2.1 & 2.49 & 2.01 & 51.7 & 0.34 & & Blanca & 20 & 12 & \\
\hline P. ligularis & 79 & 7.4 & 57.1 & 56.1 & 42.6 & 194.8 & 3.85 & 4.47 & Blanca & 27.86 & 32.3 & 0.95 \\
\hline $\begin{array}{l}P . \\
\text { quadrangularis }\end{array}$ & 1,399 & 26.37 & 71.89 & 16.5 & 6.44 & 203.8 & 0.75 & 3.75 & Amarilla & 12.78 & 151.9 & \\
\hline $\begin{array}{l}\text { P. edulis D. } \\
\text { Brasil }\end{array}$ & 81 & 6.18 & 60.5 & 46.3 & 46.5 & 197.1 & 5.07 & 3.05 & Amarilla & 19.53 & 33 & 0.34 \\
\hline
\end{tabular}

La especie que presentó el menor tamaño de fruto es $P$. foetida, pero una gran ventaja es que cuenta con un valor alto de sólidos solubles (Cuadro 3). La de mayor peso es $P$. quadrangularis, aproximadamente $1.4 \mathrm{~kg}$, por lo que esto es de esperarse ya que la correlación entre jugo y peso de fruto resulto altamente significativo, aunque los sólidos solubles son bajos (Cuadro 3). P. ligularis contó con el valor más alto de sólidos solubles, aunque cuando se quiso sembrar bajo condiciones del Campo Cotaxtla no mostro un desarrollo apropiado, debido quizás a las condiciones climáticas de la zona y por ser una especie que requiere temperaturas más bajas (Andrés et al, 2001).

\section{LITERATURA CITADA}

Andrés, A. J., Almaguer, V. G. y Santos, A. B. 2001. El cultivo d ela granada china (Passiflora ligularis Juss.). Universidad Autónoma Chapingo. Chapingo, Edo. de México. $34 \mathrm{p}$.

https://doi.org/10.5154/r.rchsh.1993.07.051

EMBRAPA. 2008. BRS Ouro Vermelho, BRS Gigante Amarelo, BRS Sol do Cerrado 
Cerrados Transferencia de tecnología. Brasil.

. García, T. M. A. 2002. Guía técnica. Cultivo de maracuyá amarillo. CENTA. El Salvador, San Salvador, 33 p.

Knight, R. J. Jr. and Sauls, J. W. 1994. The Passion fruit. Fact Sheet HS-60. UF/IFAS. 7 p.

Morton, J. F. 1987. Passionfruit Passiflora edulis Sims p. 320-328. In: Morton, J.F. (Ed). Fruits of warm climates. Creative Resources Systems Inc. Miami, Florida. USA.
Schwentesius, R. R. y Gómez, C. M. A. 1997. El Maracuyá fruta de la pasión. Universidad Autónoma Chapingo. 245 p.

Serna, V. J. 1994. El cultivo del Maracuyá. In: Memoria de la Primera Reunión Internacional y Segunda Reunión Nacional de Frutales Nativos e Introducidos. Montecillos, México. pp. 85-100.

Copyright (c) 2014 Enrique Noé Becerra Leor, Xóchitl Rosas González, Isaac Meneses Márquez y Andrés Vásquez Hernández

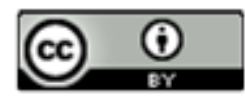

Este texto está protegido por una licencia Creative Commons 4.0.

Usted es libre para Compartir —copiar y redistribuir el material en cualquier medio of formato- y Ad aptar el documento —remezclar, transformar y crear a partir del material - para cualquier propósito, inchso para fines comerciales, siempre que cumpla la condición de:

Atribución: Usted debe dar crédito a la obra original de manera adecuada, proporcionar un enlace a la licencia, e indicar si se han realizado cambios. Puede hacerio en cualquier forma razonable, pero no de forma tal que sugiera que tiene el apoyo del licenciante olo recibe por el usoque hace de la obra.

Resumendelicencia - Textocompletodelalicencia

Revista Científica Biológico Agropecuaria Tuxpan 2 (1)

ISSN: 2007-6940 